\title{
Patient monitoring: on-line computing
}

\author{
JoHn C. A. RaISON \\ Department of Health \& Social Security, London, S.E.1
}

\begin{abstract}
Summary
On-line computing represents a further extension of the senses of attendant staff in intensive care situations.

Smaller computers will contribute as scientific processors, by extending the capability of instrumental systems or enabling new means of measurement and calculation.

The fullest use of computer-aid will provide systems of data acquisition from multiple sources, rapid processing correlation, analysis in mathematical and diagnostic forms, and resultant output in simply intelligible displays: later, some direct control of therapy may be applied.
\end{abstract}

\section{On-line computing}

The speed and flexibility of calculation, analysis and data presentation which an on-line digital computer can bring to bear make inevitable its significant contribution to the care of patients suffering from acute physiological derangements or instability. Analogue computers are already components of commercially available monitoring equipment. There is evidence that some of the more complex of these instruments provide unreliable diagnostic information (Rawles, 1969) and a plea has been made to reverse increasing complexity (Stewart, 1969).

Such viewpoints might seem to point against the development of digital or hybrid (analogue/digital) computers in intensive care. Stewart (1969) calls for a relatively limited set of correlated measurements attainable from simpler apparatus, but outlines requirement of twenty-seven coded signals for interpretation. Maloney (1968) presses the need for identification of 'rare event' phenomena in monitoring, which lies beyond the ability of present day electronic apparatus, or its likely development. Digital computers offer the means to extract more information from presently measured variables and to extend the range of these which may be tapped and correlated with others to extend the power of monitoring. The explosive development of biomedical engineering and computer technology has left the use of computers for monitoring mainly in the hands of research workers whose prime concern is still further to probe their possibilities or to acquire new pathophysiological knowledge, rather than to produce more immediately a practical and useful clinical system. There are components in each of the well established computer-based monitoring systems which could be usefully combined to provide such a system, but the options are being developed so fast that it would not be sensible to consider those which are now practical and used as examples in this review as the best for assembly in any clinical system projected for, say, 2 years ahead. Improvements in care which may be achieved by computers are characterized as those which:

(a) better the collection, display and recording of complex information (data assembly);

(b) perform tedious calculation immediately (scientific processing);

(c) undertake analytical procedures, usually of multivariables, and sometimes producing output in clinical terms (data analysis);

(d) 'close the loop' by initiating treatment in response to measurements made (process control).

These are not compartments, but rather form a spectrum of computer capability, and parts, or all of them, may contribute to an operative system.

Data acquired for such a system may be both analogue and alphanumeric. The Karolinska Hospital Thoracic Clinic system (Mellberg, 1968; Norlander, 1968; William-Olsson et al., 1969) at present receives input only from keyboards: at some terminal positions input is assisted by reference to a movable cursor on a series of predetermined cathoderay tube displays. A wide range of the normally recorded clinical variables can be entered on computer file by this means. The visual display unit (VDU) output is of a number of variable displays producing combinations of alphanumeric and graphic information. Terminals are also stationed remote from the intensive care unit (laboratory, doctor's office, etc) so that ease and speed of communication are added to the considerable aid in eliminating nurses' clerical duties provided by this mainly 'data assembly' system. It is important not to think of this merely as reducing the burden of collecting information, 
significant as this is. The clarity of the collected and displayed data and its accessibility are of notable assistance to attendant staff.

All other existing systems receive analogue data from the range of accepted biomedical transducers, usually as a voltage signal. Multiplexors are instruments which permit serial sampling, which may be very rapid, of a large number of different signals (from different physiological channels such as ECG, arterial waveform, or even different patients) by a single computer-input channel. Each such sampling is an identification of the voltage at that moment which is then quantified by an analogue-digital converter for further processing. The analogy is that a continuously written wave-form is converted to a series of isolated dots. Each 'dot', as a number, may require storage if it is to be processed. The first necessity is therefore to determine how frequently an individual signal source is to be sampled. The decision is a combination of clinical, physical and dataprocessing considerations. Temperature, for instance, may need reading only every half-hour. A signal involved in a critical alarm system may have to be read continuously, although, fortunately, almost all of the data thereby collected can normally be 'dumped' almost immediately. Other, more detailed measurement (arterial, venous pressures and respiratory characteristics) can be made every 5-10 mins. The physical characteristics of a biologically variable waveform are of significance: sampling at twice the highest frequency of waveform to be identified is often adequate, although higher rates may be needed for precise measurement of peaks and troughs. Sampling is timed by an internal computer clock, and a reading every 4.2 msecs (circa $200 / \mathrm{sec}$ ) has often been chosen. The third factor is the amount of processing required in real-time during each data acquisition period or in the interval before the subsequent period. A computer-based monitoring system works necessarily in real-time, that is, the output information is made available sufficiently quickly for appropriate action to be taken. It should also be expected to function 24 hours daily throughout the week except for minimal breaks for maintenance. It will usually be the case, therefore, that all the required processing of data, including output, must be carried out between the periods of clinical measurement (data acquisition periods) or during them (since some physiological channels will be monitored continuously). This processing time can be estimated when the extent of analysis and display programs are known. The potential of any particular computer installation is therefore a compound of the number of input channels to be sampled, sampling frequency, and the intensity of subsequent processing. Acceptable reduction in any one of these categories will increase the scope of the other two. Here it is that hybrid computer systems offer advantages. The interposition of 'black boxes', filters, delays and integrators, pulse height analysers, and other special purpose analogue preprocessors or fixed-wire computers can reduce the demand on the main digital computer. ECG analysis illustrates these points. The various programmes which provide reports comparable with those of a cardiologist (Pipberger, 1965) require high frequency sampling, and considerable processing must occur after the period of data acquisition. It is not possible to use these continuously for monitoring. Relatively simpler requirements can be set, such as rate calculation, alarm limits and counting of ectopic beats, with shorter programmes and lower frequency of sampling. Further economy can be effected by using the analogue method of Daly \& Johnson (1968). When both $x$ and $y$ axes of a vectorcardiogram exceed set limits, an ectopic beat is scored. By these means, a larger number of patients can be monitored. On the occurrence of predetermined events, a much fuller programme of sampling and diagnostics can be called into action, to identify ectopic characteristics or provide complete analysis, since these more protracted procedures are now of rare event phenomena which can be accommodated within the system. This concept offers prospects of extending an immediate diagnostic service to the at-risk patient with myocardial infarction who may have been removed, after the first few days, from an intensive care unit. Durfey \& Leeming (1966) used hybrid methods for respiratory monitoring. Computers serving the present working systems are so large that only during periods of most active data acquisition have they been strained at all, and in the intervals between they have been able to undertake new program testing, totally unrelated 'batch-processing' jobs, or have uneconomic idle processing time. But, in terms of designing an effective clinical system within an appropriate cost-limit the 'pay-off' situation existing between the various data factors and hybrid techniques must be fully explored. Few of these constraints are so important in digital computers confined to the scientific processor mode. In this, the computer performs a combination of data analogue acquisition, analysis, calculation and displayed output, usually relating to a single procedure of clinical measurement. The advantages over traditional methods are that (a) calculations are performed within seconds rather than perhaps hours and can therefore have greater importance in therapy: the measurement can be repeated much more frequently, and (b) such a processing system may permit new instrumentation or greater accuracy in method. Small, special purpose computers are already available commercially for the determination of cardiac output by dye-dilution estimation. It is possible that other 
specific small instruments may be produced, but of greater interest is the use of a small general purpose digital computer capable of several different complicated measuring methods. Such a machine may require 'recompiling' (the insertion of a new set of instructions, usually by paper tape) for each separate task, in order to keep to a minimum the 'core' size (the main processing component of the computer) for economy. The relatively small cost of these machines makes for the fastest development of clinical apparatus to be expected for computers working in this mode. It is, however, with application of the real-time, multivariable multiprocessing activity, the data analysis mode, that a digital computer offers unique facilities for monitoring. Further development of existing monitoring equipment, perhaps with small analogue computers and display or recording means, may, alone, lead to the disadvantages of need for multiple analytic modules, their complexity of arrangement round each patient, frequent moving, and the constant observation of many such discrete instruments or the interpretation of complex coded light signals. It is more appropriate to incorporate the computer in building up a monitoring system. None of the present installations provides the optimum pattern for a working model, by virtue of their emphasis on research; but a review of their facets will illustrate the clinical advantages available and the multiprocessing activities of the computer. All of them acquire nearly continuous analogue signals of ECG and arterial pressure, the latter from an intravascular catheter, usually introduced percutaneously. This is regarded as a routine, safe practice for almost all patients sufficiently ill to require complex monitoring. (Extravascular methods based on Korotkov's sounds, plethysmography or photometry are not used although a computer offers possibilities of overcoming some shortcomings of present apparatus.) By analytical and correlative programs, the computer identifies damping of the arterial catheter, eliminates the huge number of 'false alarms' generated by conventional monitoring equipment (Raison et al., 1968a) and produces simple diagnosis in words of limit-exception conditions. Central venous pressure is measured automatically, without need for repositioning of the patient, or adjustment of transducer level (Raison et al., 1968b). Measurement of temperature provides opportunity for correlations between body and skin temperature, and of both in relation to ambient temperature. The importance of peripheral skin temperature as a prognostic sign in shock has already been demonstrated (Shubin \& Weil, 1966; Joly \& Weil, 1969), and little further correlative work is required to produce other clinical aids relating to peripheral circulation and metabolic needs, from these combined sources.
Ventilatory and respiratory monitoring exemplifies the function of the computer in multivariable measurement, analysis, calculation, and correlation. Present 'manual' methods to achieve the same ends require application of several different and inconvenient pieces of apparatus, much measurement and calculation: full use is not clinically practicable.

In the system developed in San Francisco (Osborn et al., 1968) a modified pneumotachograph, which can comfortably be fitted for continuous use to the airway of any patient requiring such monitoring, is used to collect pressure and flow data as well as gas samples during each analysis period. In addition to rate estimation, ventilatory mechanics (tidal and minute volumes, compliance, non-elastic resistance and alveolar ventilation), oxygen uptake and carbon dioxide elimination are calculated on a breath-bybreath basis (Beaumont et al., 1968). The gas sample is drawn continuously through rapid $\mathrm{CO}_{2}$ and $\mathrm{O}_{2}$ electrode analysers (Elliott, Segger \& Osborn, 1966). There is a delay between production of the flow signal waveform, from which volumetric estimations are made, and the corresponding ones from the gas electrodes for each breath. The computer monitors this delay and makes appropriate correlations of data points (Raison et al., 1968c). There are then steps of multiplication, integration, averaging, subtraction of expired from inspired volumes, recalculation of gas volumes related to differences of composition of inspired and expired air (Osborn et al., 1968, 1969a). In addition, the computer system blows warm air down sampling lines between analyses, preventing condensation and thereby enabling the apparatus to remain untended for more than 24 hours. This involves control of several valves and careful volume-balancing procedures to ensure similarity of ventilation during and between analyses. Algorithms also contain analytical steps identifying falling compliance, airway obstruction or leakages, and undesirable ventilatory gas composition. Results appear within about $2 \mathrm{sec}$ of each $30 \mathrm{sec}$ analysis period. Such techniques are impossible without use of a computer. These measurements have all produced significant new physiological information about the acutely ill and direct therapeutic aids: these include simpler and better techniques of establishing and monitoring patients on ventilators (Raison, 1968d) and much earlier treatment to control scarcely noticeable shivering with its marked metabolic demands (Raison, Beaumont \& Osborne, 1968e). Of eight otherwise 'unexplained arrhythmic crises', five were of respiratory origin several hours earlier, quite capable of computer diagnosis (Osborn et al., 1969b). It must not be pretended that such automatic diagnosis is yet made, but once identified from the computer record, appropriate programming is relatively easy. Full development of the diagnostic 


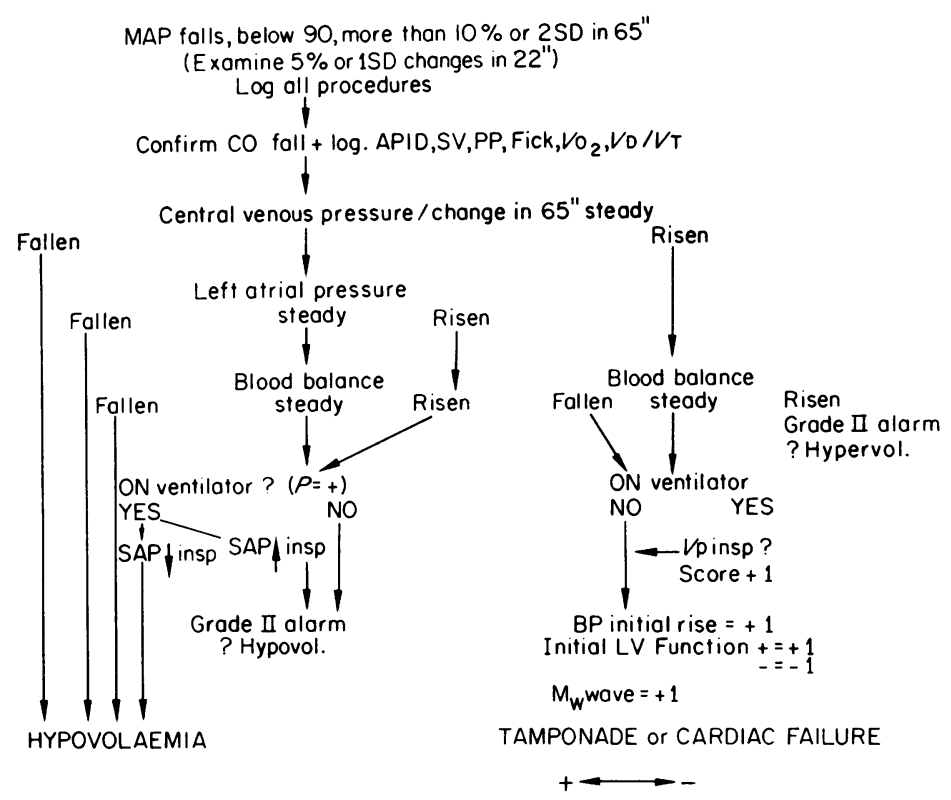

Fig. 1. A Flow chart for cardiac complications. The second procedural step refers to various methods of cardiac output $(\mathrm{CO})$ estimation or measurements correlating with changes of $\mathrm{CO}$ which are available to the system. The last procedures correlate changes in left and right atrial and arterial waveforms which have been interpreted as clinically significant with observations of ventilation. Five different variable characteristics may each initiate any of sixteen pathway courses to produce three diagnostic indications or two tentative, lesser (Grade II) alarms.

monitoring possibilities of computers, wherein lies their most important contribution to care, will only occur with their availability. Trend identification has to be brought from a state of generalization to that of quantified interrelation of the multivariables under review, with appropriate latitudes of acceptable measurement. Since this depends upon extensive surveys, often of multiple data arrays from the same clinical episode, and directed towards correlation with what are rare event phenomena, the task cannot be achieved without the flexible data handling of a computer. Fig. 1 represents an elementary version of flow-path diagram for computer differential diagnosis of three serious complications following cardiac surgery. The hypothesized correlation of measurements, based on clinical observations and laboratory experiment, requires testing under clinical conditions, and 'tuning', the selection from those suggested of the limits by which it should operate (Osborn et al., 1969a). For this purpose, the first evolved computer programme, operating on-line but as a background activity, should compile multiple records of diagnostic activity based on the various limits suggested. The system should make its own statistical analysis of the alternatives suggested, cutting out tedious perusal of nearly overwhelming quantities of digital data, for correlation with clinically defined conditions. It is impossible to anticipate such complicated procedures without computer aid. Virtually none of such diagnostic work is dependent upon major improvements in the instruments of measurement, since the system itself may be used to enhance them or minimize their defects: there is considerable new significant information to be obtained from signals now available. Research development of computer monitoring can be expected to find new pathophysiologic trends and to increase the means of measurement which can be applied comfortably and reliably: impedance pneumography is likely to be one such. Even later, monitoring by reference to computer models may be possible. A two-wavelength oximeter (Eberhart, 1968) connected on-line to a computer is combined with oxygen uptake measurement to provide cardiac output estimations (Osborn et al., 1969a). The computer also reads cardiac output dye-dilution curves. Different methods of estimation may be applied (Dow, 1955). At present much more extensive study, again of the type unlikely without computer aid, is required to identify the most useful computation in critical states. The computer offers programmed variation of the method of calculation for different conditions, if this is necessary. Warner, Gardner \& Toronto (1968) derive stroke volume changes by computation from arterial waveform, disposing of the need for special injection. Data from blood gas analysis is correlated with ventilatory gas 
data to provide alveolar-arterial oxygen gradients and ventilation/perfusion ratios. On-line measurement of blood gas and pH is now performed (Clark $\&$ Gardner, 1968), and, recently, sodium and potassium (Widdowson, G. \& Osborn, J. J., San Francisco, personal communication). Temperature, $\mathrm{pH}$ and other necessary coefficient adjustments are made by computer.

'Closing the loop' by making the computer initiate treatment resembles the process control mode of an industrial computer. Whilst there will be hesitation about this application until greater confidence in the precision of equipment and clinical decision criteria can be secured, the systems in use in Rochester, Minnesota and Birmingham, Alabama (Sheppard et al., 1968) control and monitor blood replacement by reference to arterial and venous pressures and blood loss, all measured automatically. The doctor in charge can set appropriate variables. It seems probable that more effective and safer use of the vasoactive drugs in shock can be provided by computer control and monitoring (Sheppard, 1969, personal communication). Many nurses will be relieved to be spared the chore of fluid balance chart maintenance which can be provided by such systems.

The clinical staff must be confident of the reliability of such complex systems. Precise equipment, relatively simple to use, has been developed to assist necessary routine standardization procedures by manual means: some automatic calibration is already incorporated and there are no barriers to further development. At present most systems require about 45 min setting up and rechecking by an electronics technician every $24 \mathrm{hr}$ and can then be supervised by nurses. It is valuable at present to provide the latter with a large cathode ray display of vascular and ECG waveforms which have the function of reassuring staff that the computer is measuring 'expected' biological data rather than an artificial signal. All of the described systems contain some provision for recording: some include nurses' or doctors' notes, or drug administration. Output methods have improved to the point where it is possible to indicate the optimals to be chosen. Hard copy printouts will probably be required for longterm use, since most of the disc-stored data is 'dumped' at intervals of 1-7 days. It may remain desirable to plot data graphically against a time base. A slow recorder driven by the computer allows the coincident automatic plotting of analogue data and therapeutic comments if required. For immediate use, information displays on cathode ray tubes are preferred. Most users retain flexibility of format to heighten impact on staff: graphic layouts together with no more than two or three corresponding digital values seem most effective. Information of particular significance can be presented in flashing form. In early stages of identifying new clinically significant correlations, techniques of display can be used to take advantage of man's pattern recognition ability to test different hypotheses. However, it is clear that the computer's ability to analyse and display diagnostic or therapeutic conclusions in words, rather than merely in codes, lights or digits is a major contribution to care and that an operative system should use this to the maximum. Input methods for observer data now use ten to sixteen position keyboards; choice from a series of brief input displays, arranged in a logical tree-branching manner for rapid review, permit easy entry of considerable selected data. A larger keyboard is usually provided remotely for free-form entries.

What then, is the place for computer-based monitoring systems? Since the research models already contribute to care, a service function is inevitable. Trials specifically directed to this end, and to assessment of its effects on care and manpower and the true costs, will contribute as much to immediate progress as the work of research units. From existing techniques it is possible to provide a greater degree of monitoring, more precise diagnosis and to eliminate all but a minimum of nurse calculating and charting. Acceptance and use by staff is enthusiastic, with proper introduction, so long as the basic system is not undergoing constant revision by development activities. Computer 'down time' has become so infrequent and short as not to jeopardize its clinical use. Systems are left untended by computer personnel for up to $66 \mathrm{hr}$ at weekends.

It is possible that, in time, less expensive purposebuilt computer systems executing most of the complex functions will be designed and built, in which case widespread use may become economically possible. At present, however, it is suggested that this prospect is remote, and must wait on much more precise clinical information. The viable alternative, use of small-to-medium size computers, seems at first sight formidably expensive. Sheppard (Sheppard et al., 1968) has estimated a $50 \%$ increase of charges in a 14-bedded unit, but existing research systems in America have not been designed for maximum economy in a service situation. Certainly, however, this consideration suggests that application must be in relatively large centres. Data may be collected from terminals scattered in a hospital, but ensuing costs rise, and the installation of a computer will contribute to an argument for as much centralization of various intensive units as is possible. For each terminal site, analogue computational methods, which for some functions such as integration, differentiation and logarithmic function generation are more efficient, should be used when possible, so that only reduced data essentially requiring digital processing is passed to the computer. Twenty to sixty 
terminal stations in intensive units and operating theatres seem feasible for an installation. This means also that some investigative laboratories could be supported (cardiac catheterization, pulmonary function), or there is a possibility of combination with a chemical pathological laboratory.

\section{Acknowledgments}

Most of the concepts and practical work described herein were evolved in collaboration with my colleagues, Drs J. J. Osborn, J. O. Beaumont and F. Gerbode, at the Pacific Medical Center, San Francisco. My warm appreciation of this association extends to many other members of the team. The project was supported by grant No. 5PO1-HE-06311-08 of the National Heart Institute, N.I.H., Bethesda.

\section{References}

Beaumont, J.O.B., Osborn, J.J., Raison, J.C.A. \& Russell, J.A.G. (1968) Respiratory measurement and monitoring based on an on-line computer. Proceedings of the Conference for the Association for the Advancement of Medical Instrumentation, Houston.

Clark, J.S. \& GaRdNer, R.M. (1968) On-line computerized acid-base analysis. Proceedings of the 21 st Annual Conference on Engineering in Biology and Medicine, Institute of Electrical and Electronic Engineers, New York. Houston, 17, 7.

DALY, J.J. \& Johnson S.G. (1968) Automated method for monitoring ventricular ectopic beats. Lancet, $2,813$.

Dow, P. (1955) Dimensional relationships in dye-dilution curves from human and dogs, with empirical formula for certain troublesome curves. Journal of Applied Physiology, 7, 399.

DURFeY, J.Q. \& LeEming, M.N. (1966) An on-line system for measuring respiratory parameters using a hybrid analogue/ digital computing system. Aerospace Medicine, 37, 474.

EBERHART, R.C. (1968) An automated sampling whole blood photometer. Biomedical Sciences Instrumentation, 4, 197.

Elliott, S.E., Segger, F.J. \& Osborn, J.J. (1966) A modified oxygen gauge for the rapid measurement of $\mathrm{PO}_{2}$ in respiratory gases. Journal of Applied Physiology, 21, 1672.

JoLY, H.R. \& WeIL, M.H. (1969) Temperature of the great toe as an indication of the severity of shock. Circulation, $39,131$.

MALONEY, J.V. (1968) The trouble with patient monitoring. Annals of Surgery, 168, 605.

Mellberg, K. (1968) Integrated data display system for patient monitoring at the Caroline Hospital. Proceedings of 21 st Annual Conference on Engineering in Biology and Medicine, Institute of Electrical and Electronic Engineers, New York. Houston, 17, 8.

NORLANDER, O. (1968) An integrated patient monitoring system with display terminals. Fourth World Congress of Anesthiologists. Excerpta Medica Foundation, Amsterdam.
Osborn, J.J., Beaumont, J.O., Raison, J.C.A., Russell, J. \& Gerbode, F. (1968). Measurement and monitoring of acutely ill patients by digital computer. Surgery, 64, 1057.

Osborn, J.J., Beaumont, J.O., Raison, J.C.A. \& AbBott, R. (1969a) Computation for quantitative on-line measurements in an intensive care ward. Computers in Biomedical Research, Vol. III (ed. by Stacy, R.W. \& Waxman, B.). Academic Press, New York.

Osborn, J.J., Raison, J.C.A., Beaumont, J.O., Hill, J.D. Kerth, W.J., Popper, R.W. and Gerbode, F. (1969b) Respiratory causes of 'sudden unexplained arrhythmia' in post-thoracotomy patients. Scientific sessions. 22nd Conference of the American Heart Association.

Pipberger, H.V. (1965) Computer analysis of the electrocardiogram. In Computers in Biomedical Research, 11, 377-407. Vol. I (ed. by Stacy, R.W. \& Waxman, B.). Academic Press, New York.

Raison, J.C.A., Beaumont, J.O.B., Russell, J.A.G. \& OsBoRN, J.J. (1968a) Alarms in an intensive care unit: an interim compromise. Computers and Biomedical Research, $1,556$.

Raison, J.C.A., Beaumont, J.O., Shore, J. \& Osborn, J.J. (1968b) Automated measurement of central venous pressure by on-line real-time computer method. Proceedings of the 21st Annual Conference on Engineering in Biology and Medicine, Institute of Electrical and Electronic Engineers, New York. Houston, 4, 9.

Raison, J.C.A., Beaumont, J.O., Elliott, S.E. \& Osborn J.J. (1968c) Breath-by-breath analysis of respiratory gases in acutely ill patients. Proceedings of the 21 st Annual Conference on Engineering in Biology and Medicine, Institute of Electrical and Electronic Engineers, New York. Houston, 2, 6.

RAISON, J.C.A. (1968d) Respiratory aspects of measurement and pathophysiology in shock. Conference on Dynamics of Septic Shock in Man. Albert Einstein College of Medicine and National Academy of Sciences. (To be published, Little, Brown: Boston.)

Raison, J.C.A., Beaumont, J.O. \& Osborn, J.J. (1970) Oxygen consumption after open-heart surgery measured by a digital computer system. Annals of Surgery, (In press).

RaWles, J.M. (1969) Patient monitoring: a clinician's point of view. Biomedical Engineering, 4, 264.

Sheppard, L.C., Kouchoukos, N.T., Kurtts, M.A. \& KIRKLIN, J.W. (1968) Automated treatment of critically ill patients following operation. Annals of Surgery, 168, 596

Shubin, T.H. \& WeIL, M.H. (1966) Efficient monitoring with a digital computer of cardiovascular function in seriously ill patients. Annals of Internal Medicine, 65, 453.

STEWART, J.S.S. (1969) Meaningful monitoring. Lancet, i, 1305.

WARner, H.R., Gardner, R.M. \& Toronto, A.F. (1968) Computer-based monitoring of cardiovascular functions in postoperative patients. Circulation. Supplement II, 1168.

William-olsson, G., Norlander, O., Nordent, I. \& Petterson, S.O. (1969) A patient monitoring system with display terminals. Opuscula Medica, 2, 39. 